\title{
Effect of Top-Region Area of Flat-Top Pyramid Patterned Sapphire Substrate on the Optoelectronic Performance of GaN-Based Light-Emitting Diodes
}

\author{
Hsu-Hung Hsueh, ${ }^{1}$ Sin-Liang Ou, ${ }^{2}$ Yu-Che Peng, ${ }^{1}$ Chiao-Yang Cheng, \\ Dong-Sing Wuu, ${ }^{2,4}$ and Ray-Hua Horng ${ }^{1,5}$ \\ ${ }^{1}$ Graduate Institute of Precision Engineering, National Chung Hsing University, Taichung 402, Taiwan \\ ${ }^{2}$ Department of Materials Science and Engineering, Da-Yeh University, Changhua 515, Taiwan \\ ${ }^{3}$ Wafer Works Corporation, Taoyuan 32542, Taiwan \\ ${ }^{4}$ Department of Materials Science and Engineering, National Chung Hsing University, Taichung 402, Taiwan \\ ${ }^{5}$ Department of Electronics Engineering, National Chiao Tung University, Hsinchu 300, Taiwan
}

Correspondence should be addressed to Ray-Hua Horng; rhh@nctu.edu.tw

Received 31 March 2016; Accepted 16 June 2016

Academic Editor: Wei-Heng Shih

Copyright (C) 2016 Hsu-Hung Hsueh et al. This is an open access article distributed under the Creative Commons Attribution License, which permits unrestricted use, distribution, and reproduction in any medium, provided the original work is properly cited.

\begin{abstract}
The flat-top pyramid patterned sapphire substrates (FTP-PSSs) have been prepared for the growth of GaN epilayers and the fabrication of lateral-type light-emitting diodes (LEDs) with an emission wavelength of approximately $470 \mathrm{~nm}$. Three kinds of FTP-PSSs, which were denoted as FTP-PSS-A, FTP-PSS-B, and FTP-PSS-C, respectively, were formed through the sequential wet etching processes. The diameters of circle areas on the top regions of these three FTP-PSSs were 1, 2, and $3 \mu \mathrm{m}$, respectively. Based on the X-ray diffraction results, the full-width at half-maximum values of rocking curves at (002) plane for the GaN epilayers grown on conventional sapphire substrate (CSS), FTP-PSS-A, FTP-PSS-B, and FTP-PSS-C were 412, 238, 346, and 357 arcsec, while these values at (102) plane were 593, 327, 352, and $372 \mathrm{arcsec}$, respectively. The SpeCLED-Ratro simulation results reveal that the LED prepared on FTP-PSS-A has the highest light extraction efficiency than that of the other devices. At an injection current of $350 \mathrm{~mA}$, the output powers of LEDs fabricated on CSS, FTP-PSS-A, FTP-PSS-B, and FTP-PSS-C were 157, 254, 241, and 233 mW, respectively. The results indicate that both the crystal quality of GaN epilayer and the light extraction of LED can be improved via the use of FTP-PSS, especially for the FTP-PSS-A.
\end{abstract}

\section{Introduction}

Recently, GaN-based materials have received a lot of attention for many optoelectronic applications consisting of lightemitting diodes (LEDs), laser diodes, and high-power devices [1-3]. Because the price of bulk GaN substrate is very high, the GaN-based epilayers should be prepared on foreign substrates, such as sapphire and silicon carbide. At present, sapphire is the most common substrate for the epitaxial growth of GaN-based LEDs. However, owing to the large mismatch in lattice constant between GaN and sapphire, a large number of threading dislocations (TDs) generated during the epitaxial growth process would lead to a serious degradation of the GaN crystal quality. For the fabrication of GaN-based LEDs, the deterioration of epitaxial quality will obviously limit their optoelectronic performances including internal quantum efficiency (IQE), electron mobility, and device lifetime.

Thus, to reduce the TD density of GaN epitaxial film, several techniques have been presented [4-6]. Among these techniques, the epitaxial lateral overgrowth (ELOG) employing the stripe-mask patterns on the $\mathrm{GaN}$ epilayer is a useful method to obstruct the propagation of TDs efficiently, resulting in a significant reduction of TD density [4]. Nevertheless, when the ELOG is performed, the growth interruption caused by the mask deposition is inevitable. Additionally, 


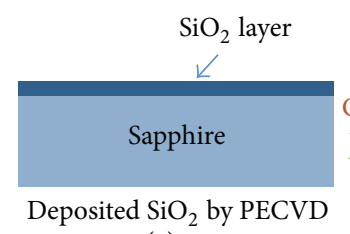

(a)

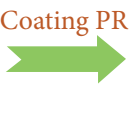

(1)

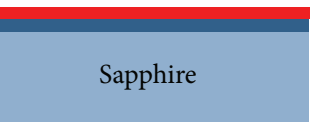

(b)

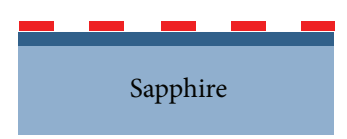

(c)

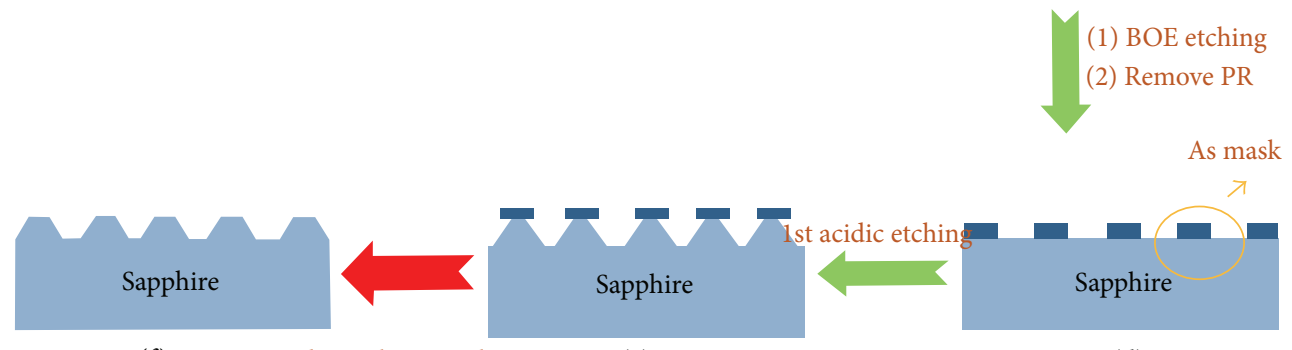

(f) Removed LTO by HF solution

(e)

FIGURE 1: Fabrication processes of FTP-PSS: (a) growth of a $\mathrm{SiO}_{2}$ layer on the $c$-plane sapphire by PECVD, (b) preparation of a thick PR on the $\mathrm{SiO}_{2}$ layer by spin-coating, (c) performing the photolithography technique to open the $\mathrm{PR}$ mask, (d) formation of the round-shaped $\mathrm{SiO}_{2}$ layer via the buffered oxide etching and removal of the $\mathrm{PR}$ mask, (e) wet etching to the sapphire substrate using a mixed $\mathrm{H}_{2} \mathrm{SO}_{4}: \mathrm{H}_{3} \mathrm{PO}_{4}(3: 1)$ solution at $250^{\circ} \mathrm{C}$ for $30 \mathrm{~min}$, and (f) removal of the $\mathrm{SiO}_{2}$ mask layer using a $\mathrm{HF}$ solution.

the formation of mask could also induce the impurity contamination in the subsequent epilayers. To overcome these mask-related drawbacks, the mask-free patterned sapphire substrate (PSS) technology has been proposed and becomes very promising for the growth of high-quality GaN epilayer [7-10]. Except for the improvement in the IQE, the use of PSS also can increase the light extraction efficiency (LEE) of LEDs due to the nonplanar interface between sapphire and GaN.

In previous researches, the InGaN/GaN film with high crystal quality can be achieved using metalorganic chemical vapor deposition (MOCVD) on the flat-top pyramidal PSS (FTP-PSS) [8-10]. These researches confirm that the FTPPSSs are indeed helpful to enhance the performance of LEDs. However, the influence of pattern size of the FTP-PSS on the device characteristics is seldom studied. In our work, three FTP-PSSs were fabricated to serve as the substrates for the growth of GaN-based epitaxial structures. In these three FTP-PSSs, the heights of patterns are equal to each other. Meanwhile, the diameters of circle areas on both the top and the bottom regions of the flat-top pyramidal patterns in these FTP-PSSs are modified. The effect of the flat-top region area of FTP-PSS on the device performance was investigated.

\section{Experimental}

The fabrications of PSSs with the flat-top pyramid patterns were completed through the wet etching processes sequentially, as described in Figure 1. As shown in Figure 1(a), the $\mathrm{SiO}_{2}$ layer with a thickness of $250 \mathrm{~nm}$ was firstly prepared on the $c$-plane sapphire by plasma enhanced chemical vapor deposition (PECVD). Then, a thick photoresist (PR) was grown on the $\mathrm{SiO}_{2}$ layer as a mask by spin-coating (Figure $1(\mathrm{~b})$ ). To open the PR mask, the photolithography technique was performed on the PR layer (Figure 1(c)). In
Figure $1(\mathrm{~d})$, the round-shaped $\mathrm{SiO}_{2}$ layer was prepared using a buffered oxide etch (BOE) solution to serve as a mask for the fabrication of PSS, and the PR mask was removed. As shown in Figure 1(e), the sapphire substrate with the $\mathrm{SiO}_{2}$ mask was etched using a mixed $\mathrm{H}_{2} \mathrm{SO}_{4}: \mathrm{H}_{3} \mathrm{PO}_{4}(3: 1)$ solution at $250^{\circ} \mathrm{C}$ for $30 \mathrm{~min}$. In this research, three different diameters $(1,2$, and $3 \mu \mathrm{m}$ ) of round shape were designed for the $\mathrm{SiO}_{2}$ mask layers. Thus, after removing the $\mathrm{SiO}_{2}$ mask layer using a $\mathrm{HF}$ solution (Figure 1(f)), the FTP-PSSs with three different topregion areas can be formed.

Except for the FTP-PSSs used to fabricate the LEDs, the LED on the conventional sapphire substrate (CSS) was also prepared as the contrasted sample. The LED epitaxial structures grown on FTP-PSSs and CSS were performed by low-pressure MOCVD at the same growth conditions. The LED epitaxial structure consisted of a $3 \mu \mathrm{m}$ thick undoped GaN (u-GaN) layer, a $2 \mu \mathrm{m}$ thick n-type GaN:Si layer, 11 periods of InGaN/GaN multiple quantum wells (MQW), a $100 \mathrm{~nm}$ thick p-type AlGaN layer, and a $0.2 \mu \mathrm{m}$ thick p-type GaN:Mg layer. At the beginning of epitaxial growth, it is noted that a $30 \mathrm{~nm}$ thick GaN buffer layer was prepared at low temperature $\left(530^{\circ} \mathrm{C}\right)$, and then a $3 \mu \mathrm{m}$ thick $\mathrm{u}-\mathrm{GaN}$ layer was deposited at high temperature $\left(1150^{\circ} \mathrm{C}\right)$. After growing these epilayers, the device processes for lateral-type LED were carried out subsequently. Firstly, a mesa pattern with the size of $45 \mathrm{mil} \times 45 \mathrm{mil}$ was defined and prepared via the standard photolithography and dry etching processes. Then, the ITO film was grown on the p-type GaN layer to serve as a transparent conducting layer. The ITO film was deposited by E-beam evaporation at $270^{\circ} \mathrm{C}$, and its thickness was $200 \mathrm{~nm}$. Finally, the $\mathrm{Ti} / \mathrm{Al} / \mathrm{Ti} / \mathrm{Au}(15 / 2000 / 25 / 60 \mathrm{~nm})$ metal layers grown on the sample by thermal evaporation were adopted as both $n$-pad and p-pad electrodes. These LEDs all possess the emission wavelength approximately $470 \mathrm{~nm}$. 


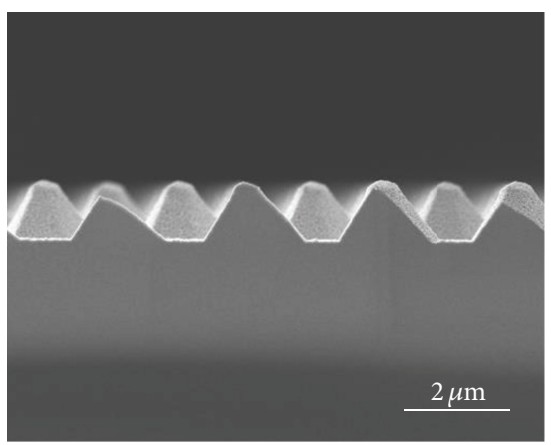

(a)

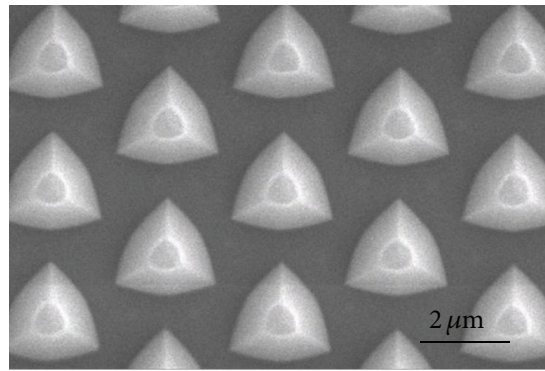

(d)

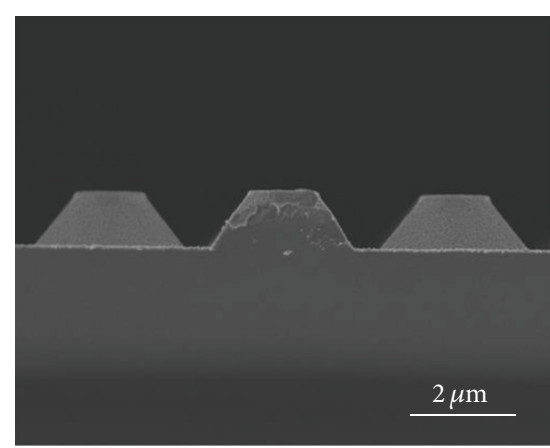

(b)

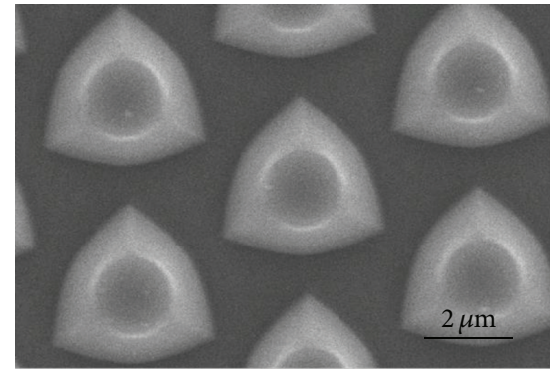

(e)

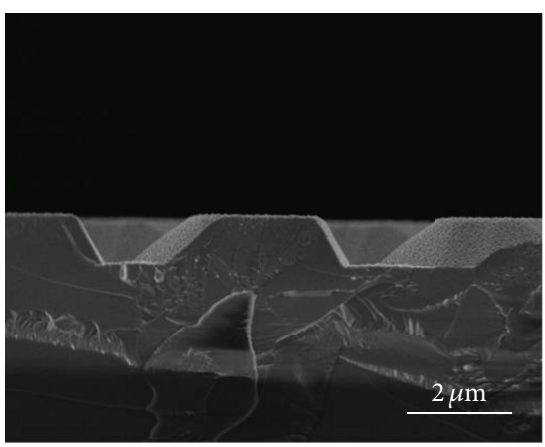

(c)

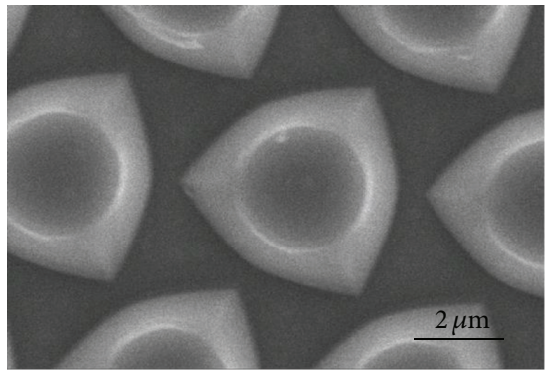

(f)

Figure 2: Cross-sectional SEM image of (a) FTP-PSS-A, (b) FTP-PSS-B, and (c) FTP-PSS-C. Top-view SEM images of (d) FTP-PSS-A, (e) FTP-PSS-B, and (f) FTP-PSS-C.

Crystal quality of the u-GaN epilayer was determined by X-ray diffraction (XRD, Philips X'Pert MRD). Here, the analyses of (002) and (102) planes for the $\mathrm{u}-\mathrm{GaN}$ epilayer were both performed. The surface morphology of the FTP-PSS was observed by scanning electron microscopy (SEM, JEOL JSM7001F). Current-voltage $(I-V)$ characteristics of these LEDs were investigated by a semiconductor parameter analyzer (Keithley, 2400 SourceMeter), and the output powers were measured with a calibrated integrating sphere. To quantitatively characterize the light extraction efficiency of LEDs on these sapphires, the simulation of photon trajectory was performed by SpeCLED-Ratro software (commercial lightsimulation software based on geometrical optics).

\section{Results and Discussion}

As mentioned above, during the fabrication process of FTPPSSs, three $\mathrm{SiO}_{2}$ mask layers with various diameters of 1,2 , and $3 \mu \mathrm{m}$ of round shape were prepared. Then, three various FTP-PSSs, that were denoted as FTP-PSS-A, FTP-PSS-B, and FTP-PSS-C, respectively, were formed via the sequential wet etching processes. Figures 2(a), 2(b), and 2(c) show the cross-sectional SEM images of FTP-PSS-A, FTP-PSS-B, and FTP-PSS-C, respectively. We can observe that the heights of patterns of these three FTP-PSSs were all fixed at $1.4 \mu \mathrm{m}$. Figures 2(d) and 2(e) show the top-view SEM images of these three FTP-PSSs, respectively. The diameters of circle areas on the top and bottom regions of FTP-PSS-A, FTP-PSS$\mathrm{B}$, and FTP-PSS-C were 1 and $2.4 \mu \mathrm{m}, 2$ and $3.8 \mu \mathrm{m}$, and 3 and $5.2 \mu \mathrm{m}$, respectively. Meanwhile, the distances between patterns of these three FTP-PSSs were $0.85,0.85$, and $0.75 \mu \mathrm{m}$, respectively. Obviously, the area of flat-top region for these three FTP-PSSs decreased in the following order: FTP-PSS-C $>$ FTP-PSS-B $>$ FTP-PSS-A. This indicates that the FTP-PSS$C$ has the largest $c$-plane area of flat-top region, while the FTPPSS-A possesses the smallest one among these three samples.

The crystal qualities of the $\mathrm{u}-\mathrm{GaN}$ epilayers grown on CSS and FTP-PSSs were estimated by measuring the full-width at half-maximum (FWHM) values derived from the XRD rocking curves. The XRD rocking curves obtained for the symmetric $\mathrm{GaN}(002)$ plane and the asymmetric $\mathrm{GaN}(102)$ plane of these four samples are presented in Figures 3(a) and $3(\mathrm{~b})$, respectively. By growing the u-GaN epilayer on the CSS, the FWHM value at the (002) plane was 412 arcsec, while the FWHM value at the (102) plane was 593 arcsec. Noted that the FWHMs at (002) and (102) of CSS have a little large as compared with those of previously reports [11, 12]. It is well know that the crystal quality of the epilayer is dependent on the MOCVD system. In this study, the growth parameters of CSS have been optimized. On the other hand, the FWHM values at the (002) plane for the u-GaN epilayers grown on FTP-PSS-A, FTP-PSS-B, and FTP-PSS-C were 238, 346, and 357 arcsec, respectively. Additionally, the FWHM values at the (102) plane for the $\mathrm{u}-\mathrm{GaN}$ epilayers prepared on these three FTP-PSSs were measured to be 327, 352, and 372 arcsec, respectively. It is well known that crystal quality of the (002) plane was influenced by screw and mixed dislocations, while the crystal quality of (102) plane was influenced by edge, screw, and mixed dislocations [13]. It is clear that the crystallinity of $\mathrm{u}-\mathrm{GaN}$ epilayer grown on the FTP-PSS is much higher than that on the CSS. This can be attributed to the reduction in the dislocation density of $\mathrm{GaN}$ epilayer 


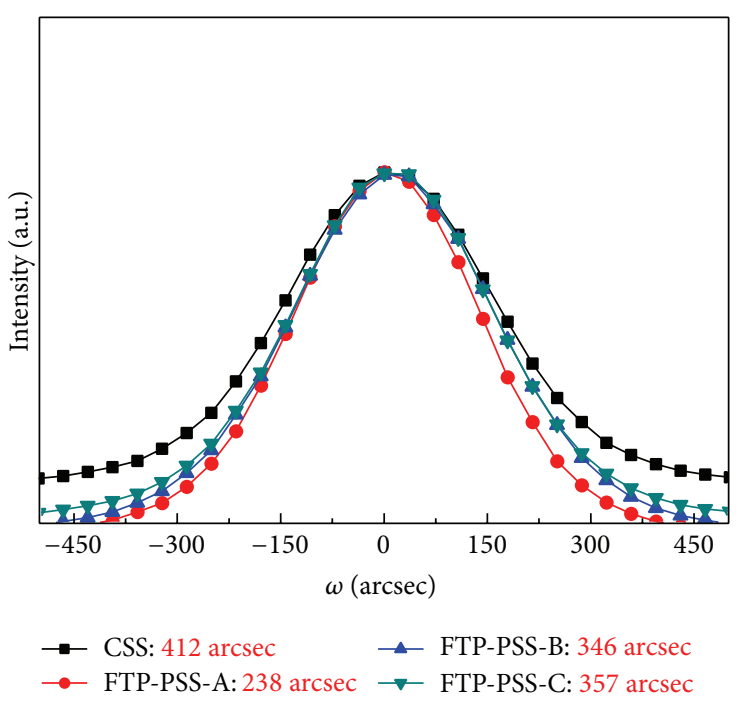

(a)

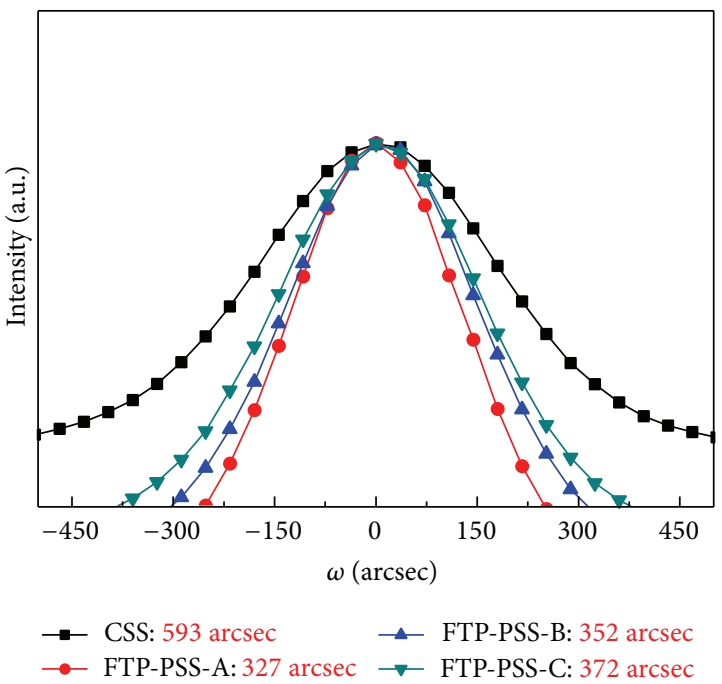

(b)

FIGURE 3: XRD rocking curves obtained for (a) the symmetric GaN(002) plane and (b) the asymmetric GaN(102) plane of GaN epilayers grown on CSS and various FTP-PSSs.

grown on the FTP-PSS. Obviously, the fabrication of FTP-PSS is useful to enhance the crystal quality of GaN epilayer.

Except for the XRD FWHM value, the evaluation of dislocation density also can be used to analyze the crystal quality of $\mathrm{u}-\mathrm{GaN}$ epilayer. The TD characteristics in $\mathrm{u}-\mathrm{GaN}$ films on CSS and FTP-PSSs were investigated by etch-pit-density (EPD) measurements. The etching process was performed by soaking the samples into the $\mathrm{H}_{2} \mathrm{SO}_{4}: \mathrm{H}_{3} \mathrm{PO}_{4}(3: 1)$ mixed solution at $250^{\circ} \mathrm{C}$ for $10 \mathrm{~min}$. Figures $4(\mathrm{a}), 4(\mathrm{~b}), 4(\mathrm{c})$, and $4(\mathrm{~d})$ display the SEM images for the TDs of $\mathrm{u}-\mathrm{GaN}$ epilayers on CSS, FTP-PSS-A, FTP-PSS-B, and FTP-PSS-C, respectively. It can be found that the EPDs of these four samples were estimated to be $3.25 \times 10^{7}, 1.39 \times 10^{6}, 2.31 \times 10^{6}$, and $5.17 \times$ $10^{6} \mathrm{~cm}^{-2}$, respectively. As marked in Figures 4(a) and 4(b), the feature of the screw dislocation is a well-defined hexagonal shape with a large black core, while the mixed dislocation has an unclear hexagonal shape with a small black core. Besides, the edge dislocation shows an unclear polygon [12, 14]. It was obvious that three various types of TDs all can be identified in the GaN epilayer on the CSS (Figure 4(a)). After growing the GaN epilayer on the FTP-PSS, the formation of these three types of TDs reduced efficiently. Moreover, the suppressions of screw and mixed TDs are more obvious than that of edge TDs. Based on XRD and EPD results, the dislocation density of GaN epilayer on the FTP-PSS can be decreased apparently as compared with that on CSS. As the FTP-PSSs were used, the GaN growth could primarily occur in the bottom and flat-top regions (they are both $c$-plane), and the epitaxial lateral overgrowth generated to merge the GaN films $[15,16]$, resulting in the decrease in the dislocation density.

Figures 5(a)-5(c) display the schematics of initial GaN growth modes on FTP-PSS-A, FTP-PSS-B, and FTP-PSS-C, respectively [17]. As discussed in Figure 2, the FTP-PSS-C possessed a largest pattern size, while the FTP-PSS-A had a smallest pattern size. Moreover, the distances between patterns of these three FTP-PSSs were similar to each other. These reveal that the number of patterns per unit area reduced in the following order: FTP-PSS-A > FTP-PSS-B > FTP-PSS$C$. In other words, the area of inclined region in these three PSSs per unit area also decreased in the following order: FTPPSS-A > FTP-PSS-B > FTP-PSS-C. As described above, a $30 \mathrm{~nm}$ thick $\mathrm{GaN}$ buffer layer was grown at $530^{\circ} \mathrm{C}$, and then the growth temperature was increased to $1150^{\circ} \mathrm{C}$. During the heating process, the thin GaN film was gradually transferred to $\mathrm{GaN}$ grains. When the subsequent growth was performed at high temperature $\left(1150^{\circ} \mathrm{C}\right)$, the $\mathrm{GaN}$ epilayer was easily deposited from these GaN grains. Because the FTP-PSS-A possessed the largest area of inclined region (per unit area) compared to that of the other two PSSs, the GaN grains were easily formed in the inclined regions during the heating process. Therefore, the GaN epilayer was easily grown on the inclined regions compared to the bottom $c$-plane regions, as shown in Figure 5(a). However, as shown in Figures 5(b) and 5(c), when the area of inclined region (per unit area) became smaller in FTP-PSS-B and FTP-PSS-C, the initial GaN growth was gradually transferred to $\mathrm{GaN}$ on $c$-plane region mode. On the other hand, in the initial growth, the GaN epilayer was also formed on the flat-top region of PSS. Since the FTP-PSS$\mathrm{C}$ had the largest flat-top region area, the GaN film was easily grown on its flat-top regions. On the contrary, the GaN film was more difficultly formed on the flat-top regions of FTPPSS-A.

The schematics of dislocation behaviors in the GaN epilayers grown on FTP-PSS-A, FTP-PSS-B, and FTP-PSS$\mathrm{C}$ were shown in Figures 5(d)-5(f), respectively [17]. As discussed above, the heights of patterns for these three FTP-PSSs were the same. Meanwhile, the distances between patterns in these three FTP-PSSs were similar to each other. Therefore, the difference in the crystal quality of GaN epilayers grown on FTP-PSS-A, FTP-PSS-B, and FTP-PSS-C could be influenced 


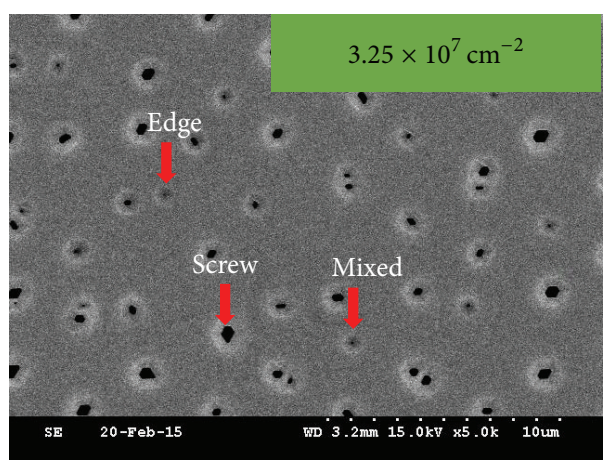

(a)

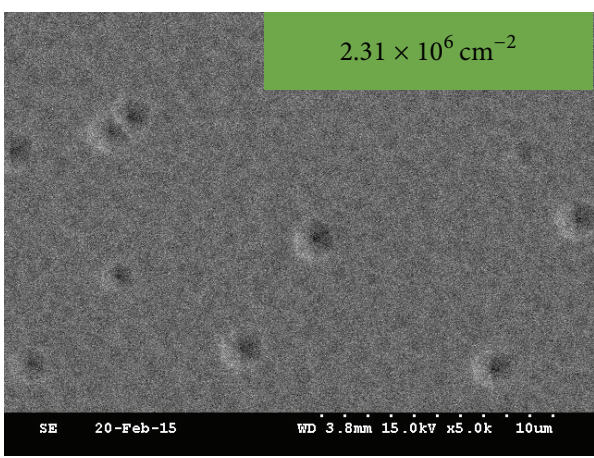

(c)

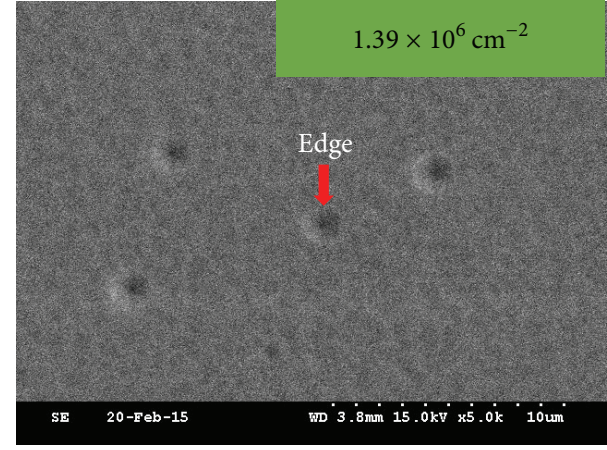

(b)

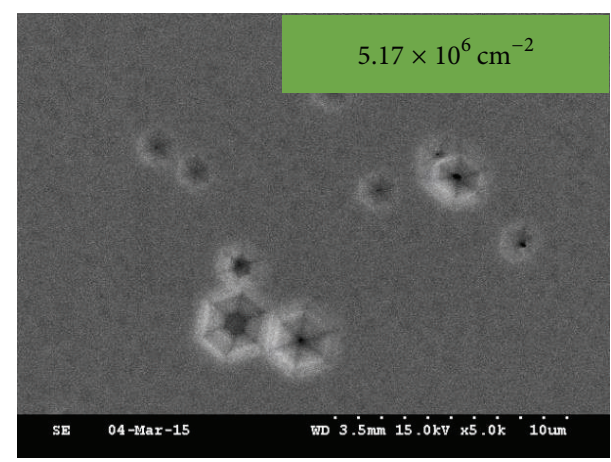

(d)

FIgURE 4: SEM images of u-GaN epilayers grown on (a) CSS, (b) FTP-PSS-A, (c) FTP-PSS-B, and (d) FTP-PSS-C after soaking the samples into the $\mathrm{H}_{2} \mathrm{SO}_{4}: \mathrm{H}_{3} \mathrm{PO}_{4}(3: 1)$ mixed solution at $250^{\circ} \mathrm{C}$ for $10 \mathrm{~min}$.

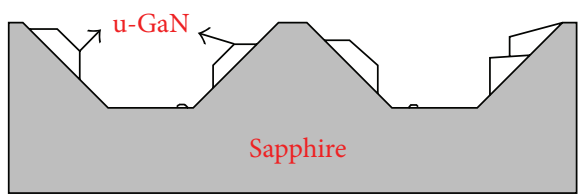

(a)

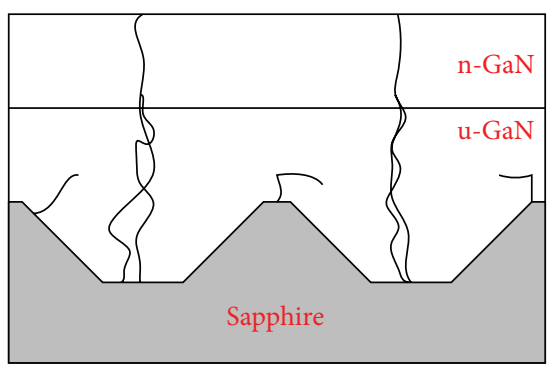

(d)

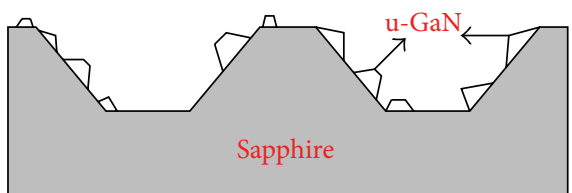

(b)

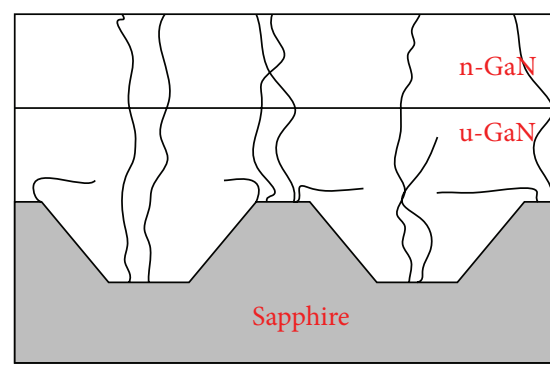

(e)

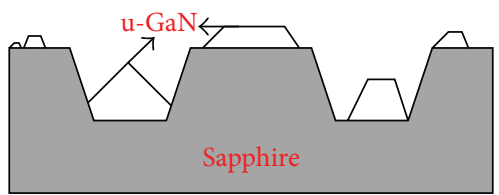

(c)

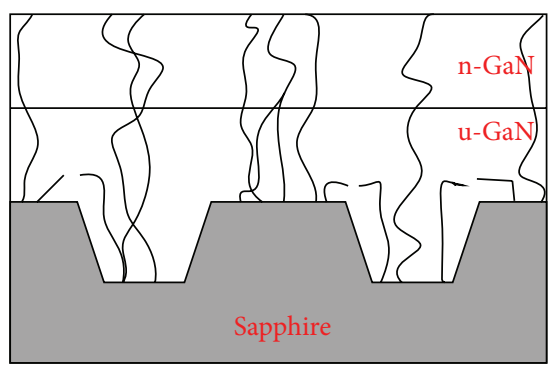

(f)

FIGURE 5: Schematic illustrations of the initial GaN growth modes on (a) FTP-PSS-A, (b) FTP-PSS-B, and (c) FTP-PSS-C. Schematic of the evolutions of dislocation behaviors in the GaN epilayers grown on (d) FTP-PSS-A, (e) FTP-PSS-B, and (f) FTP-PSS-C.

by the flat-top region area. Because the FTP-PSS-A possesses the smallest $c$-plane area of flat-top region, the number of dislocations formed at the initial stage is the least, causing the highest crystal quality of GaN epilayer grown on this PSS. On the contrary, due to the largest $c$-plane area of flat-top region formed in the FTP-PSS-C, the GaN epilayer prepared on this
PSS has a relatively low crystal quality as compared with that on the other PSSs.

Figure 6 shows the SpeCLED-Ratro simulation results for the LEDs. During the simulation, a $350 \mathrm{~mW}$ power was used to emit randomly from the MQW region. As shown in Figure 6(a), the simulated output power and LEE of the LED 

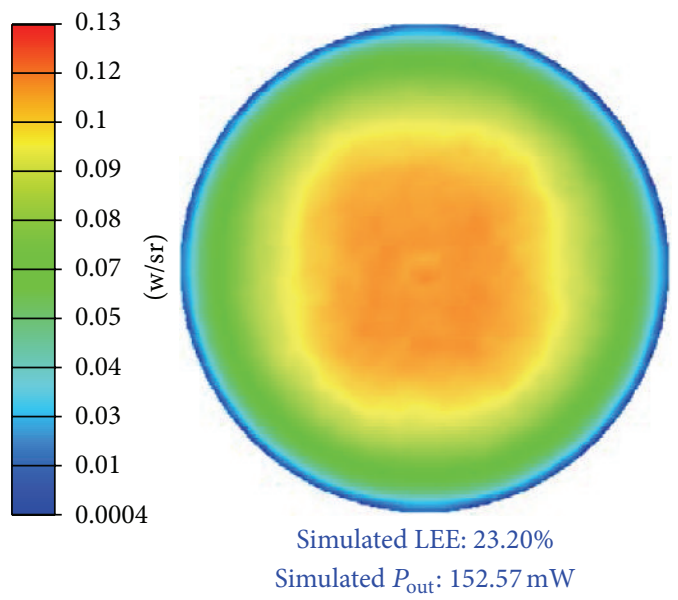

(a) LED on CSS
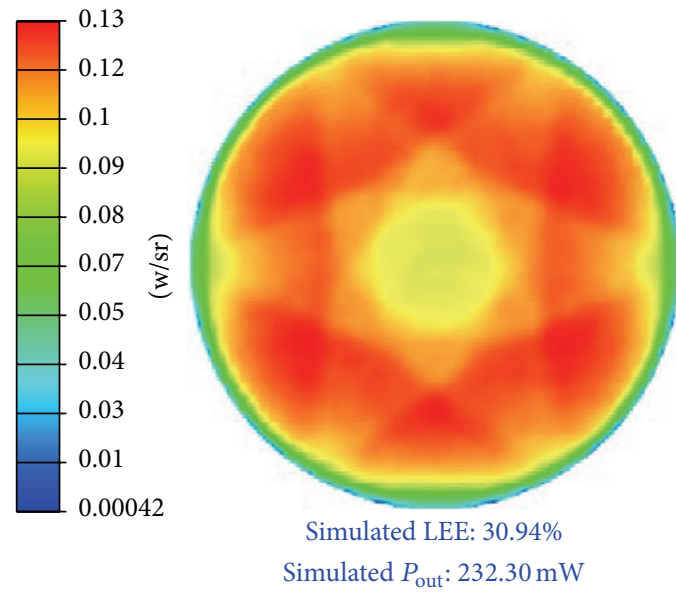

(c) LED on FTP-PSS-B
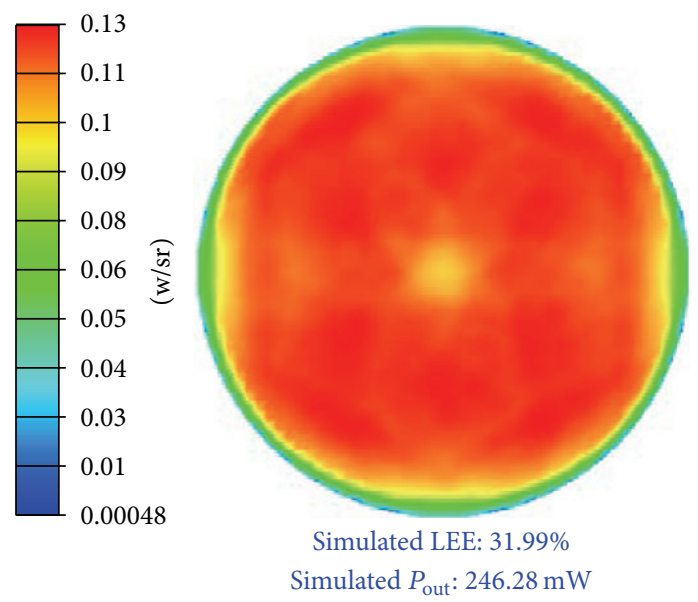

(b) LED on FTP-PSS-A
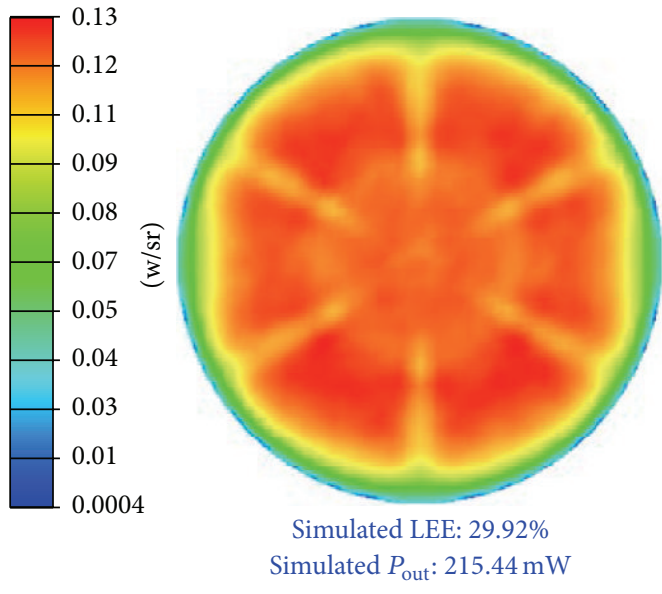

(d) LED on FTP-PSS-C

Figure 6: SpeCLED-Ratro simulation results for the LEDs fabricated on (a) CSS, (b) FTP-PSS-A, (c) FTP-PSS-B, and (d) FTP-PSS-C.

on CSS were $153 \mathrm{~mW}$ and $23.2 \%$, respectively. The simulation results for the LEDs prepared on various FTP-PSSs are shown in Figures 6(b)-6(d). We can observe that the simulated output powers of LEDs on FTP-PSS-A, FTP-PSS-B, and FTPPSS-C were increased to 246, 232, and $215 \mathrm{~mW}$; meanwhile, the simulated LEEs of these LEDs were enhanced to $32.0 \%$, $30.9 \%$, and $29.9 \%$, respectively. The results present that the LED prepared on the FTP-PSS-A possesses the highest LEE than that of the other samples.

Figure 7 displays the $I-V$ characteristics of the LEDs fabricated on CSS, FTP-PSS-A, FTP-PSS-B, and FTP-PSS-C. Although the forward voltages of these four devices measured at the whole injection currents $(0-1050 \mathrm{~mA})$ are similar to each other, there is a slight difference in the forward voltages of LEDs fabricated on CSS and FTP-PSSs. At an injection current of $350 \mathrm{~mA}$, the forward voltages of the LEDs prepared on the CSS, FTP-PSS-A, FTP-PSS-B, and FTP-PSS-C are measured to be $3.27,3.22,3.25$, and $3.27 \mathrm{~V}$, respectively. Further increasing the injection current to $1050 \mathrm{~mA}$, the forward voltages of these four devices were 4.30, 4.19, 4.26, and $4.28 \mathrm{~V}$, respectively. This indicates that the crystal quality of GaN epilayer also affects the $I-V$ characteristic of LED. Apparently, the higher the crystal quality is obtained in the GaN epilayer, the lower the forward voltage can be achieved in the LED.

Figure 8 shows the light output powers as a function of injection current for these four LED samples fabricated on the CSS and various FTP-PSSs. The light emission images (at $20 \mathrm{~mA}$ ) of these four LEDs are shown in the inset. Obviously, the light emission intensity of LED on FTP-PSS is much higher than that on CSS. The output powers (at $350 \mathrm{~mA}$ ) of LEDs prepared on CSS, FTP-PSS-A, FTP-PSS-B, and FTP-PSS-C were 157, 254, 241, and $233 \mathrm{~mW}$, respectively. In comparison to the LED on CSS, there are $61.8 \%, 53.5 \%$, and $48.4 \%$ enhancements in the output power (at $350 \mathrm{~mA}$ ) for the devices on FTP-PSS-A, FTP-PSS-B, and FTP-PSS$\mathrm{C}$, respectively. As the injection current was increased to $1050 \mathrm{~mA}$, the output powers of these four LEDs were 371, 581,543 , and $505 \mathrm{~mW}$, respectively. Based on the abovementioned results, the use of FTP-PSS can both enhance the 


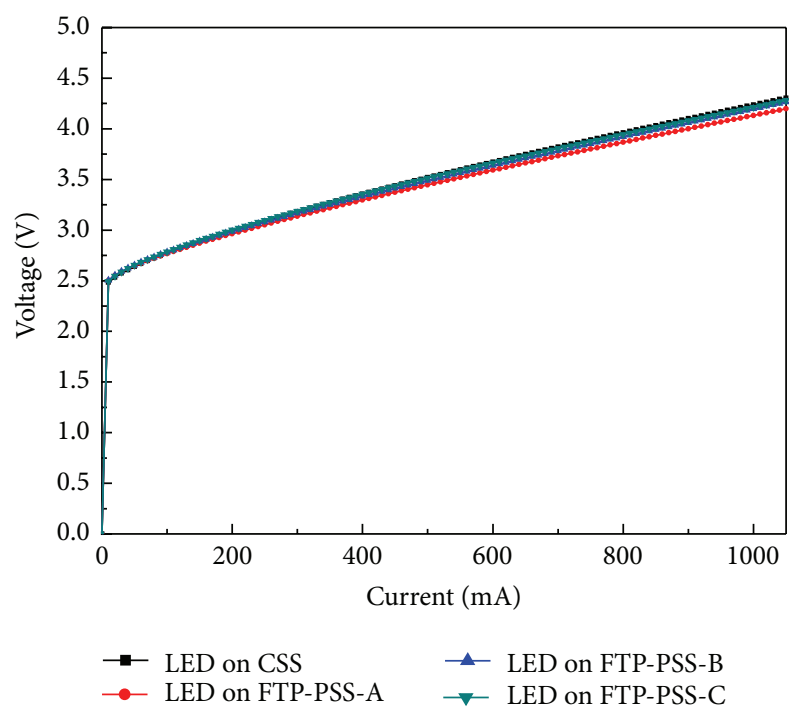

FIGURE 7: $I-V$ characteristics of the LEDs fabricated on CSS and various FTP-PSSs.

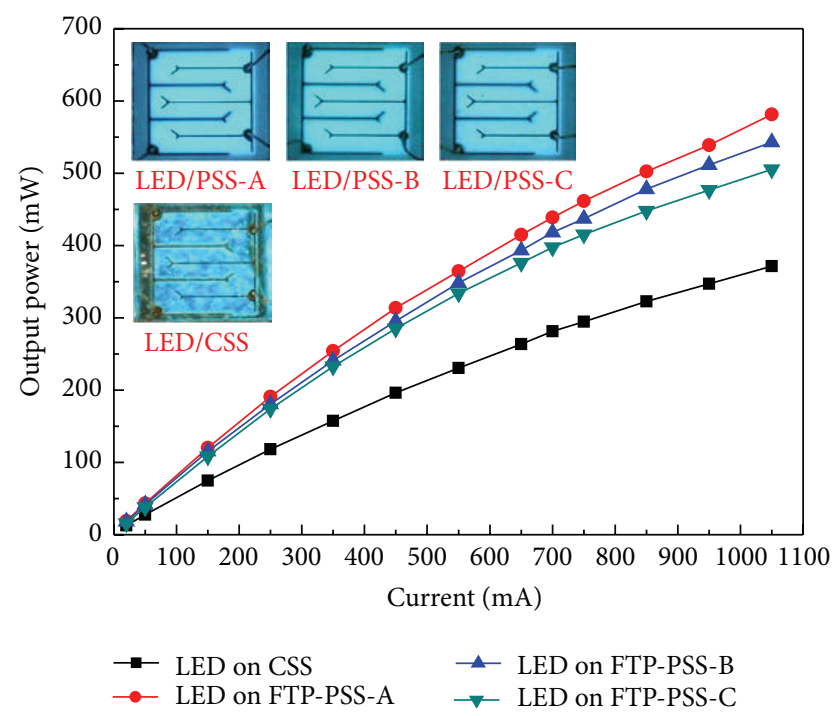

FIGURE 8: Light output powers as a function of injection current for the LEDs fabricated on CSS and various FTP-PSSs.

epilayer quality and increase the light extraction of LED. In particular, for the LED fabricated on FTP-PSS-A, it reveals that this device has better optoelectronic performances.

\section{Conclusion}

In summary, by performing the sequential wet etching processes, the FTP-PSSs have been prepared for the fabrication of GaN-based blue LEDs. The influence of the flat-top region area of FTP-PSS on the LED performance was analyzed. After fabricating the LEDs on FTP-PSSs, the optoelectronic performance of device was enhanced remarkably as compared with that prepared on CSS. Particularly, for the use of FTP-PSS-A, the crystal quality of $\mathrm{GaN}$ epilayer and the light extraction in the LED were both improved most obviously. At an injection current of $350 \mathrm{~mA}$, the output power of LEDs prepared on CSS was measured to be $157 \mathrm{~mW}$. Additionally, as the LEDs fabricated on FTP-PSS-A, FTP-PSS-B, and FTP-PSS-C, the output powers (at $350 \mathrm{~mA}$ ) were increased to 254, 241, and $233 \mathrm{~mW}$, respectively. Compared with the LED on CSS, there is $61.8 \%$ increment in the output power (at $350 \mathrm{~mA}$ ) for the devices on FTP-PSS-A.

\section{Competing Interests}

The authors declare that they have no competing interests.

\section{Acknowledgments}

This work was supported by the Ministry of Science and Technology of Taiwan under Grant no. 102-2221-E-009-196MY3, Ministry of Economic Affairs under Contract no. 100EC-17-A-07-S1-158, 102-E0605, and Hsinchu Science Park under Contracts nos. 103A13 and 104A23.

\section{References}

[1] S.-L. Jeng, C.-C. Wu, and W.-H. Chieng, "Gallium nitride electrical characteristics extraction and uniformity sorting," Journal of Nanomaterials, vol. 2015, Article ID 478375, 15 pages, 2015.

[2] G. Wang, X. Duan, and W. Chen, "Barrier thickness and hydrostatic pressure effects on hydrogenic impurity states in wurtzite $\mathrm{GaN} / \mathrm{Al}_{x} \mathrm{Ga}_{1-x} \mathrm{~N}$ strained quantum dots," Journal of Nanomaterials, vol. 2015, Article ID 937310, 9 pages, 2015.

[3] P. C. Chang, K. H. Lee, Z. H. Wang, and S. J. Chang, "AlGaN/GaN high electron mobility transistors with multi$\mathrm{Mg}_{x} \mathrm{~N}_{y}$ /GaN Buffer," Journal of Nanomaterials, vol. 2014, Article ID 623043, 4 pages, 2014.

[4] J. Han, M. H. Crawford, R. J. Shul et al., "AlGaN/GaN quantum well ultraviolet light emitting diodes," Applied Physics Letters, vol. 73, no. 12, pp. 1688-1690, 1998.

[5] A. Y. Polyakov, N. B. Smirnov, E. B. Yakimov, I.-H. Lee, and S. J. Pearton, "Electrical, luminescent, and deep trap properties of Si doped n-GaN grown by pendeo epitaxy," Journal of Applied Physics, vol. 119, no. 1, Article ID 015103, 2016.

[6] K. Hiramatsu, K. Nishiyama, M. Onishi et al., "Fabrication and characterization of low defect density GaN using facetcontrolled epitaxial lateral overgrowth (FACELO)," Journal of Crystal Growth, vol. 221, no. 1-4, pp. 316-326, 2000.

[7] C.-C. Kao, Y.-K. Su, C.-L. Lin, and J.-J. Chen, "The aspect ratio effects on the performances of GaN-based light-emitting diodes with nanopatterned sapphire substrates," Applied Physics Letters, vol. 97, no. 2, Article ID 023111, 2010.

[8] R.-M. Lin, Y.-C. Lu, S.-F. Yu et al., "Enhanced extraction and efficiency of blue light-emitting diodes prepared using two-step-etched patterned sapphire substrates," Journal of the Electrochemical Society, vol. 156, no. 11, pp. H874-H876, 2009.

[9] J.-H. Cheng, Y. S. Wu, W.-C. Liao, and B.-W. Lin, "Improved crystal quality and performance of GaN-based light-emitting diodes by decreasing the slanted angle of patterned sapphire," Applied Physics Letters, vol. 96, no. 5, Article ID 051109, 2010.

[10] H. Gao, F. Yan, Y. Zhang, J. Li, Y. Zeng, and G. Wang, "Improvement of the performance of GaN-based LEDs grown on sapphire substrates patterned by wet and ICP etching," SolidState Electronics, vol. 52, no. 6, pp. 962-967, 2008. 
[11] D. S. Wuu, W. K. Wang, K. S. Wen et al., "Defect reduction and efficiency improvement of near-ultraviolet emitters via laterally overgrown GaN on a GaN/patterned sapphire template," Applied Physics Letters, vol. 89, no. 16, Article ID 161105, 2006.

[12] J.-H. Lee, J. T. Oh, Y. C. Kim, and J.-H. Lee, "Stress reduction and enhanced extraction efficiency of GaN-based LED grown on cone-shape-patterned sapphire," IEEE Photonics Technology Letters, vol. 20, no. 18, pp. 1563-1565, 2008.

[13] B. Heying, X. H. Wu, S. Keller et al., "Role of threading dislocation structure on the $\mathrm{x}$-ray diffraction peak widths in epitaxial GaN films," Applied Physics Letters, vol. 68, no. 5, pp. 643-645, 1996.

[14] T. Hino, S. Tomiya, T. Miyajima, K. Yanashima, S. Hashimoto, and M. Ikeda, "Characterization of threading dislocations in GaN epitaxial layers," Applied Physics Letters, vol. 76, no. 23, pp. 3421-3423, 2000.

[15] L. Lu, Z. Y. Gao, B. Shen et al., "Microstructure and origin of dislocation etch pits in $\mathrm{GaN}$ epilayers grown by metal organic chemical vapor deposition," Journal of Applied Physics, vol. 104, no. 12, Article ID 123525, 2008.

[16] H. Lahrèche, P. Vennéguès, B. Beaumont, and P. Gibart, "Growth of high-quality GaN by low-pressure metal-organic vapour phase epitaxy (LP-MOVPE) from 3D islands and lateral overgrowth," Journal of Crystal Growth, vol. 205, no. 3, pp. 245252, 1999.

[17] H.-C. Lin, H.-H. Liu, G.-Y. Lee et al., "Effects of lens shape on GaN grown on microlens patterned sapphire substrates by metallorganic chemical vapor deposition," Journal of the Electrochemical Society, vol. 157, no. 3, pp. H304-H307, 2010. 

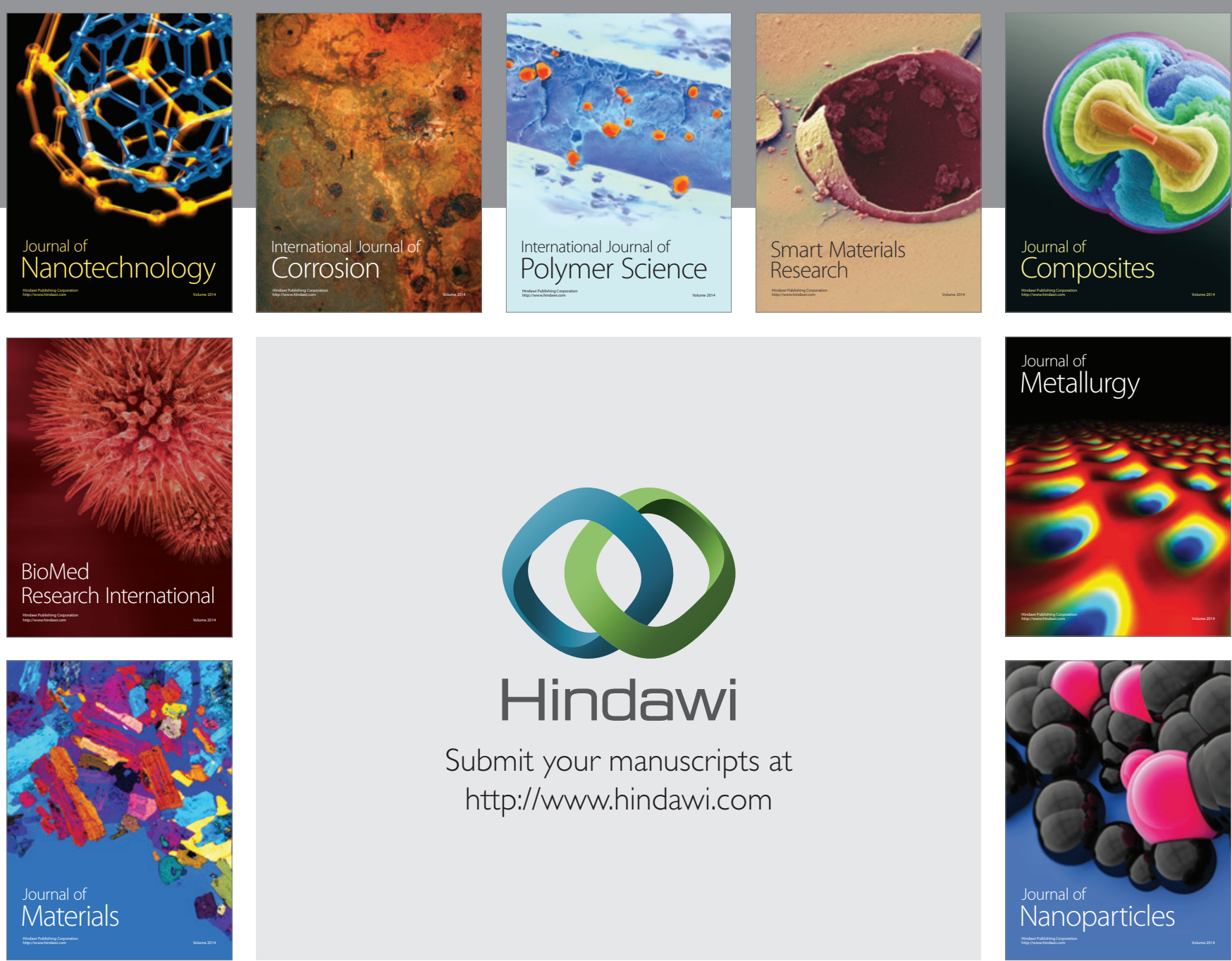

\section{Hindawi}

Submit your manuscripts at

http://www.hindawi.com

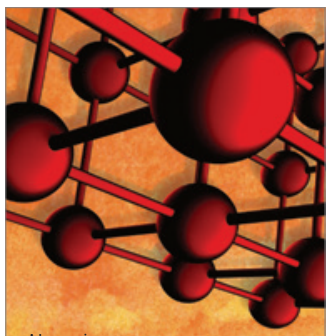

Materials Science and Engineering
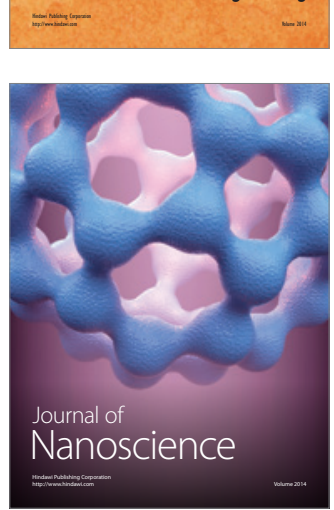
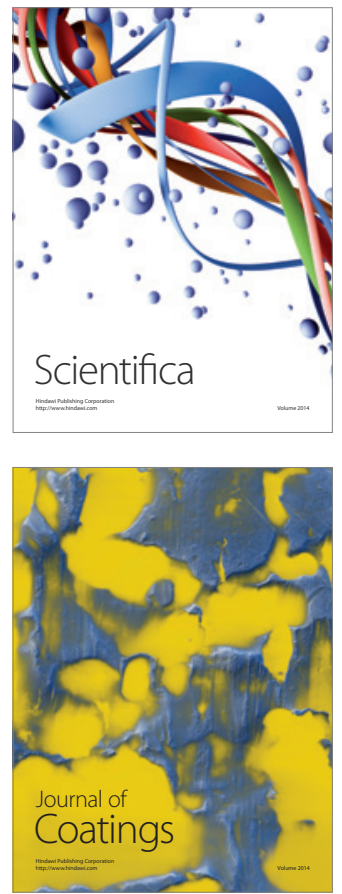
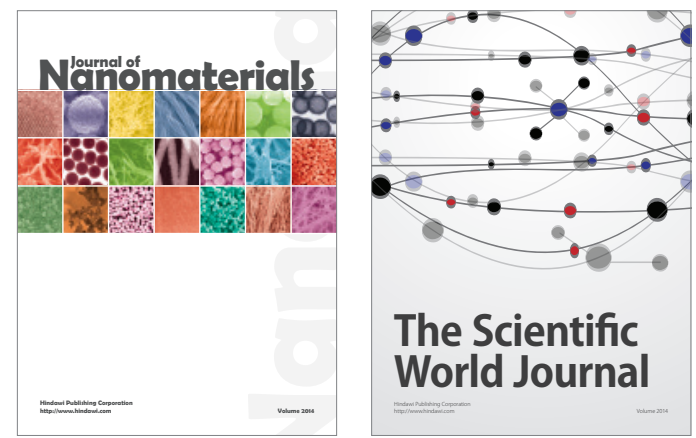

The Scientific World Journal
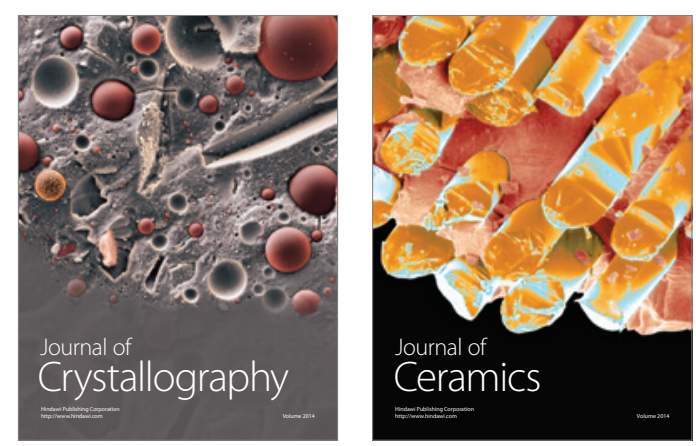
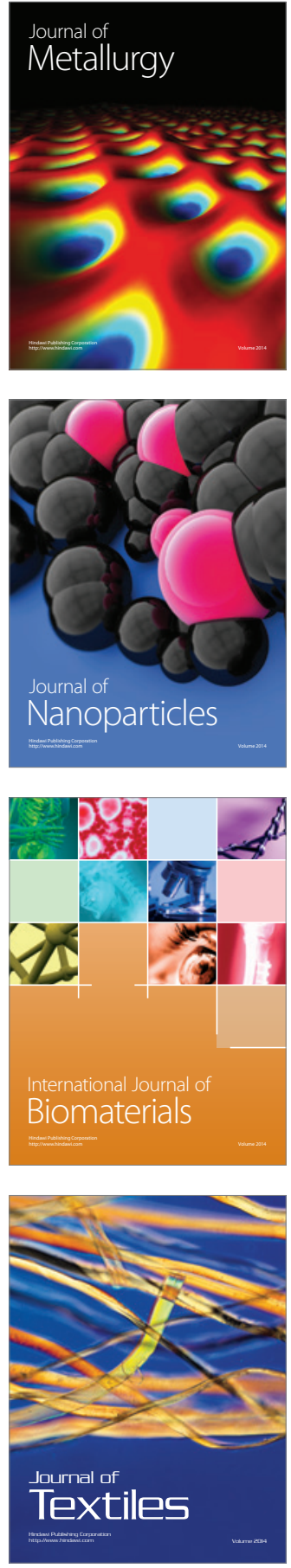